\title{
CONTROLLABILITY OF STOCHASTIC IMPULSIVE NEUTRAL FUNCTIONAL DIFFERENTIAL EQUATIONS DRIVEN BY FRACTIONAL BROWNIAN MOTION WITH INFINITE DELAY
}

\author{
EL HASSAN LAKHEL
}

Cadi Ayyad University, National School of Applied Sciences, 46000 Safi, Morocco

\begin{abstract}
In this paper we study the controllability results of impulsive neutral stochastic functional differential equations with infinite delay driven by fractional Brownian motion in a real separable Hilbert space. The controllability results are obtained using stochastic analysis and a fixed-point strategy. Finally, an illustrative example is provided to demonstrate the effectiveness of the theoretical result.
\end{abstract}

Keywords: Controllability, impulsive neutral functional differential equations, fractional powers of closed operators, infinite delay, fractional Brownian motion.

AMS Subject Classification: 35R10, 93B05 60G22, 60H20.

\section{INTRODUCTION}

The notion of controllability is of great importance in mathematical control theory. Many fundamental problems of control theory such as pole-assignment, stabilizability and optimal control my be solved under the assumption that the system is controllable. The problem of controllability is to show the existence of control function, which steers the solution of the system from its initial state to final state, where the initial and final states may vary over the entire space. Conceived by Kalman, the controllability concept has been studied extensively in the fields of finite-dimensional systems, infinite-dimensional systems, hybrid systems, and behavioral systems. If a system cannot be controlled completely then different types of controllability can be defined such as approximate, null, local null and local approximate null controllability. For more details the reader may refer to [11, 12, 21, 22, 23] and the references therein. In this paper, we study the controllability of neutral functional stochastic differential equations of the form

$$
\begin{gathered}
d\left[x(t)-g\left(t, x_{t}\right)\right]=\left[A x(t)+f\left(t, x_{t}\right)+B u(t)\right] d t+\sigma(t) d B^{H}(t), t \in[0, T], \\
\\
\left.\Delta x\right|_{t=t_{k}}=x\left(t_{k}^{+}\right)-x\left(t_{k}^{-}\right)=I_{k}\left(x\left(t_{k}^{-}\right)\right), k=1, \ldots, m, \\
x(t)=\varphi(t) \in L_{2}^{0}\left(\Omega, \mathcal{B}_{h}\right), \text { for a.e. } t \in(-\infty, 0] .
\end{gathered}
$$

Here, $A$ is the infinitesimal generator of an analytic semigroup of bounded linear operators, $(S(t))_{t \geq 0}$, in a Hilbert space $X ; B^{H}$ is a fractional Brownian motion with $H>\frac{1}{2}$ on a real and separable Hilbert space $Y$; and the control function $u(\cdot)$ takes values in $L^{2}([0, T], U)$, the Hilbert space of admissible control functions for a separable Hilbert space $U$; and $B$ is a bounded linear operator from $U$ into $X$. 
The history $x_{t}:(-\infty, 0] \rightarrow X, x_{t}(\theta)=x(t+\theta)$, belongs to an abstract phase space $\mathcal{B}_{h}$ defined axiomatically, and $f, g:[0, T] \times \mathcal{B}_{h} \rightarrow X$ are appropriated functions, and $\sigma:[0, T] \rightarrow \mathcal{L}_{2}^{0}(Y, X)$, are appropriate functions, where $\mathcal{L}_{2}^{0}(Y, X)$ denotes the space of all $Q$-Hilbert-Schmidt operators from $Y$ into $X$ (see section 2 below). Moreover, the fixed moments of time $t_{k}$ satisfy $0<t_{1}<t_{2}<\ldots<t_{m}<T$; $x\left(t_{k}^{-}\right)$and $x\left(t_{k}^{+}\right)$represent the left and right limits of $x(t)$ at time $t_{k}$ respectively. $\Delta x\left(t_{k}\right)$ denotes the jump in the state $x$ at time $t_{k}$ with $I():. X \longrightarrow X$ determining the size of the jump.

The theory of impulsive differential equations as much as neutral partial differential equations has become an important area of investigation in recent years stimulated by their numerous applications to problems arising in mechanics, medicine and biology, economics, electronics and telecommunication etc., in which sudden and abrupt changes occur ingenuously, in the form of impulses. For more details on this theory and application see the papers [1, 15, 17, 24, 25].

It is known that fractional Brownian motion, with Hurst parameter $H \in(0,1)$, is a generalization of Brownian motion and it reduces to a standard Brownian motion when $H=\frac{1}{2}$. A general theory for the infinite-dimensional stochastic differential equations driven by a fractional Brownian motion ( $\mathrm{fBm}$ ) has begun to recieve attention by various researchers, see e.g. [2, 14, 20]. For example, Dung studied the existence and uniqueness of impulsive stochastic Volterra integro-differential equation driven by $\mathrm{fBm}$ in [8] . Using the Riemann-Stieltjes integral, Boufoussi et al. [3] proved the existence and uniqueness of a mild solution to a related problem and studied the dependence of the solution on the initial condition in infinite dimensional space. Very recently, Caraballo and Diop [6], Caraballo et al. [7], and Boufoussi and Hajji [4] have discussed the existence, uniqueness and exponential asymptotic behavior of mild solutions by using the Wiener integral.

To the best of the author's knowledge, an investigation concerning the controllability for impulsive neutral stochastic differential equations with infinite delay of the form (1.1) driven by a fractional Brownian motion has not yet been conducted. Thus, we will make the first attempt to study such problem in this paper. Our results are motivated by those in [13, 15] where the controllability of mild solutions to neutral stochastic functional integrodifferential equations driven by fractional Brownian motion with finite delays are studied.

The outline of this paper is as follows: In Section 2 we introduce some notations, concepts, and basic results about fractional Brownian motion, the Wiener integral defined in general Hilbert spaces, phase spaces and properties of analytic semigroups and the fractional powers associated to its generator. In Section 3, we derive the controllability of impulsive neutral stochastic differential systems driven by a fractional Brownian motion. Finally, in Section 4, we conclude with an example to illustrate the applicability of the general theory.

\section{PRELiminaries}

We collect some notions, concepts and lemmas concerning the Wiener integral with respect to an infinite dimensional fractional Brownian, and we recall some basic results about analytical semigroups and fractional powers of their infinitesimal generators, which will be used throughout the whole of this chapter. For details of the topics addressed in this section, we refer the reader to [18, 19] and the references therein.

Let $\left(\Omega, \mathcal{F},\left\{\mathcal{F}_{t}\right\}_{t \geq 0}, \mathbb{P}\right)$ be a complete probability space satisfying the usual conditions, meaning that the filtration is a right-continuous increasing family and $\mathcal{F}_{0}$ contains all $\mathrm{P}$-null sets. 
Consider a time interval $[0, T]$ with arbitrary fixed horizon $T$ and let $\left\{\beta^{H}(t): t \in\right.$ $[0, T]\}$ be a one-dimensional fractional Brownian motion with Hurst parameter $H \in(1 / 2,1)$. By definition, $\beta^{H}$ is a centered Gaussian process with covariance function

$$
R_{H}(s, t)=\frac{1}{2}\left(t^{2 H}+s^{2 H}-|t-s|^{2 H}\right) .
$$

Moreover, $\beta^{H}$ has the following Wiener integral representation:

$$
\beta^{H}(t)=\int_{0}^{t} K_{H}(t, s) d \beta(s),
$$

where $\beta=\{\beta(t): t \in[0, T]\}$ is a Wiener process and kernel $K_{H}(t, s)$ is the kernel given by

$$
K_{H}(t, s)=c_{H} s^{\frac{1}{2}-H} \int_{s}^{t}(u-s)^{H-\frac{3}{2}} u^{H-\frac{1}{2}} d u,
$$

for $t>s$, where $c_{H}=\sqrt{\frac{H(2 H-1)}{g\left(2-2 H, H-\frac{1}{2}\right)}}$ and $g(\cdot, \cdot \cdot)$ denotes the Beta function. We take $K_{H}(t, s)=0$ if $t \leq s$.

We will denote by $\mathcal{H}$ the reproducing kernel Hilbert space of the $\mathrm{fBm}$. Precisely, $\mathcal{H}$ is the closure of set of indicator functions $\left\{1_{[0 ; t]}: t \in[0, T]\right\}$ with respect to the scalar product

$$
\left\langle 1_{[0, t]}, 1_{[0, s]}\right\rangle_{\mathcal{H}}=R_{H}(t, s) .
$$

The mapping $1_{[0, t]} \rightarrow \beta^{H}(t)$ can be extended to an isometry between $\mathcal{H}$ and the first Wiener chaos and we will denote by $\beta^{H}(\varphi)$ the image of $\varphi$ by the previous isometry.

Recall that for $\psi, \varphi \in \mathcal{H}$, the scalar product in $\mathcal{H}$ is given by

$$
\langle\psi, \varphi\rangle_{\mathcal{H}}=H(2 H-1) \int_{0}^{T} \int_{0}^{T} \psi(s) \varphi(t)|t-s|^{2 H-2} d s d t .
$$

Consider the operator $K_{H}^{*}$ from $\mathcal{H}$ to $L^{2}([0, T])$ defined by

$$
\left(K_{H}^{*} \varphi\right)(s)=\int_{s}^{T} \varphi(r) \frac{\partial K_{H}}{\partial r}(r, s) d r .
$$

The proof of the fact that $K_{H}^{*}$ is an isometry between $\mathcal{H}$ and $L^{2}([0, T])$ can be found in [18]. Moreover, for any $\varphi \in \mathcal{H}$, we have

$$
\beta^{H}(\varphi)=\int_{0}^{T}\left(K_{H}^{*} \varphi\right)(t) d \beta(t) .
$$

It follows from [18] that the elements of $\mathcal{H}$ may be not functions but rather distributions of negative order. In order to obtain a space of functions contained in $\mathcal{H}$, we consider the linear space $|\mathcal{H}|$ generated by the measurable functions $\psi$ such that

$$
\|\psi\|_{|\mathcal{H}|}^{2}:=\alpha_{H} \int_{0}^{T} \int_{0}^{T}|\psi(s)\|\psi(t)\| s-t|^{2 H-2} d s d t<\infty,
$$

where $\alpha_{H}=H(2 H-1)$. We have the following lemma (see [18]).

Lemma 2.1. The space $|\mathcal{H}|$ is a Banach space with the norm $\|\psi\|_{|\mathcal{H}|}$; the following inclusions hold

$$
\mathbb{L}^{2}([0, T]) \subseteq \mathbb{L}^{1 / H}([0, T]) \subseteq|\mathcal{H}| \subseteq \mathcal{H} ;
$$

and for any $\varphi \in \mathbb{L}^{2}([0, T])$,

$$
\|\psi\|_{|\mathcal{H}|}^{2} \leq 2 H T^{2 H-1} \int_{0}^{T}|\psi(s)|^{2} d s .
$$


Let $X$ and $Y$ be two real, separable Hilbert spaces and let $\mathcal{L}(Y, X)$ be the space of bounded linear operator from $Y$ to $X$. For convenience, we shall use the same notation to denote the norms in $X, Y$ and $\mathcal{L}(Y, X)$. Let $Q \in \mathcal{L}(Y, Y)$ be an operator defined by $Q e_{n}=\lambda_{n} e_{n}$ with finite trace $\operatorname{tr} Q=\sum_{n=1}^{\infty} \lambda_{n}<\infty$, where $\lambda_{n} \geq 0(n=1,2 \ldots)$ are nonnegative real numbers and $\left\{e_{n}: n=1,2 \ldots\right\}$ is a complete orthonormal basis in $Y$. Let $B^{H}=\left(B^{H}(t)\right)$ be a $Y$ - valued fbm on $(\Omega, \mathcal{F}, \mathbb{P})$ with covariance $Q$ given by

$$
B^{H}(t)=B_{Q}^{H}(t)=\sum_{n=1}^{\infty} \sqrt{\lambda_{n}} e_{n} \beta_{n}^{H}(t)
$$

where $\beta_{n}^{H}$ are real, independent fBm's. This process is Gaussian, it starts from 0 , has zero mean, and covariance:

$$
E\left\langle B^{H}(t), x\right\rangle\left\langle B^{H}(s), y\right\rangle=R(s, t)\langle Q(x), y\rangle \text { for all } x, y \in Y \text { and } t, s \in[0, T] .
$$

In order to define Wiener integrals with respect to the $Q$-fBm, we introduce the space $\mathcal{L}_{2}^{0}:=\mathcal{L}_{2}^{0}(Y, X)$ of all $Q$-Hilbert-Schmidt operators $\psi: Y \rightarrow X$. Recall that $\psi \in \mathcal{L}(Y, X)$ is called a $Q$-Hilbert-Schmidt operator if

$$
\|\psi\|_{\mathcal{L}_{2}^{0}}^{2}:=\sum_{n=1}^{\infty}\left\|\sqrt{\lambda_{n}} \psi e_{n}\right\|^{2}<\infty
$$

and that the space $\mathcal{L}_{2}^{0}$ equipped with the inner product $\langle\varphi, \psi\rangle_{\mathcal{L}_{2}^{0}}=\sum_{n=1}^{\infty}\left\langle\varphi e_{n}, \psi e_{n}\right\rangle$ is a separable Hilbert space.

Let $\phi:[0, T] \rightarrow \mathcal{L}_{2}^{0}(Y, X)$ be a given function. The Wiener integral of $\phi$ with respect to $B^{H}$ is defined by

$$
\int_{0}^{t} \phi(s) d B^{H}(s)=\sum_{n=1}^{\infty} \int_{0}^{t} \sqrt{\lambda_{n}} \phi(s) e_{n} d \beta_{n}^{H}(s)=\sum_{n=1}^{\infty} \int_{0}^{t} \sqrt{\lambda_{n}}\left(K_{H}^{*}\left(\phi e_{n}\right)(s) d \beta_{n}(s),\right.
$$

where $\beta_{n}$ is the standard Brownian motion used to define $\beta_{n}^{H}$ as in (2.1).

We conclude this subsection by stating the following result which is critical in the proof of our result. It can be proved using arguments similar to those used to prove Lemma 2 in [7].

Lemma 2.2. If $\psi:[0, T] \rightarrow \mathcal{L}_{2}^{0}(Y, X)$ satisfies $\int_{0}^{T}\|\psi(s)\|_{\mathcal{L}_{2}^{0}}^{2} d s<\infty$, then (2.2) is well-defined as an $X$-valued random variable and

$$
\mathbb{E}\left\|\int_{0}^{t} \psi(s) d B^{H}(s)\right\|^{2} \leq 2 H t^{2 H-1} \int_{0}^{t}\|\psi(s)\|_{\mathcal{L}_{2}^{0}}^{2} d s .
$$

It is known that the study of theory of differential equation with infinite delays depends on a choice of the abstract phase space. We assume that the phase space $\mathcal{B}_{h}$ is a linear space of functions mapping $(-\infty, 0]$ into $X$, endowed with a norm $\|\cdot\|_{\mathcal{B}_{h}}$. We shall introduce some basic definitions, notations and lemma which are used in this paper. First, we present the abstract phase space $\mathcal{B}_{h}$. Assume that $h:(-\infty, 0] \longrightarrow[0,+\infty)$ is a continuous function with $l=\int_{-\infty}^{0} h(s) d s<+\infty$.

We define the abstract phase space $\mathcal{B}_{h}$ by

$$
\begin{aligned}
\mathcal{B}_{h}= & \left\{\psi:(-\infty, 0] \longrightarrow X \text { for any } \tau>0,\left(\mathbb{E}\|\psi\|^{2}\right)^{\frac{1}{2}}\right. \text { is bounded and measurable } \\
& \text { function on } \left.[-\tau, 0] \text { and } \int_{-\infty}^{0} h(t) \sup _{t \leq s \leq 0}\left(\mathbb{E}\|\psi(s)\|^{2}\right)^{\frac{1}{2}} d t<+\infty\right\}
\end{aligned}
$$

If we equip this space with the norm

$$
\|\psi\|_{\mathcal{B}_{h}}:=\int_{-\infty}^{0} h(t) \sup _{t \leq s \leq 0}\left(\mathbb{E}\|\psi\|^{2}\right)^{\frac{1}{2}} d t
$$


then it is clear that $\left(\mathcal{B}_{h},\|\cdot\|_{\mathcal{B}_{h}}\right)$ is a Banach space.

We now consider the space $\mathcal{B}_{D I}$ ( $D$ and $I$ stand for delay and impulse, respectively ) given by

$$
\begin{aligned}
\mathcal{B}_{D I} & =\left\{x:(-\infty, T] \rightarrow X: x \mid I_{k} \in \mathcal{C}\left(I_{k}, X\right) \text { and } x\left(t_{k}^{+}\right), x\left(t_{k}^{-}\right)\right. \text {exist with } \\
& \left.x\left(t_{k}^{-}\right)=x\left(t_{k}\right), k=1,2, \ldots, m, x_{0}=\varphi \in \mathcal{B}_{h} \text { and } \sup _{0 \leq t \leq T} \mathbb{E}\left(\|x(t)\|^{2}\right)<\infty\right\},
\end{aligned}
$$

where $x \mid I_{k}$ is the restriction of $x$ to the interval $I_{k}=\left(t_{k}, t_{k+1}\right], k=1,2, \ldots, m$. The function $\|\cdot\|_{\mathcal{B}_{D I}}$ to be a semi-norm in $\mathcal{B}_{D I}$, it is defined by

$$
\|x\|_{\mathcal{B}_{D I}}=\left\|x_{0}\right\|_{\mathcal{B}_{h}}+\sup _{0 \leq t \leq T}\left(\mathbb{E}\left(\|x(t)\|^{2}\right)\right)^{\frac{1}{2}} .
$$

The following lemma is a common property of phase spaces.

Lemma 2.3. [16] Suppose $x \in \mathcal{B}_{D I}$, then for all $t \in[0, T], x_{t} \in \mathcal{B}_{h}$ and

$$
l\left(\mathbb{E}\|x(t)\|^{2}\right)^{\frac{1}{2}} \leq\left\|x_{t}\right\|_{\mathcal{B}_{h}} \leq l \sup _{0 \leq s \leq t}\left(\mathbb{E}\|x(s)\|^{2}\right)^{\frac{1}{2}}+\left\|x_{0}\right\|_{\mathcal{B}_{h}},
$$

where $l=\int_{-\infty}^{0} h(s) d s<\infty$.

Definition 2.4. The map $f:[0, T] \times \mathcal{B}_{h} \rightarrow X$ is said to be an $L^{2}$-Carathéodory if

(1) $t \longmapsto f(t, x)$ is measurable for each $x \in \mathcal{B}_{h}$,

(2) $x \longmapsto f(t, x)$ is continuous for almost all $t \in[0, T]$,

(3) for every positive integer $q$ there exists $h_{q} \in L^{1}\left([0, T], \mathbb{R}^{+}\right)$, such that

$$
\mathbb{E}\|f(t, x)\|^{2} \leq h_{q}(t), \text { for all }\|x\|_{\mathcal{B}_{h}}^{2} \leq q \text { and for a.e. } t \in[0, T] .
$$

Next, we introduce some notations and basic facts about the theory of semigroups and fractional power operators. Let $A: D(A) \rightarrow X$ be the infinitesimal generator of an analytic semigroup, $(S(t))_{t \geq 0}$, of bounded linear operators on $X$. The theory of strongly continuous is thoroughly discussed in [19] and [9]. It is well-known that there exist $M \geq 1$ and $\lambda \in \mathbb{R}$ such that $\|S(t)\| \leq M e^{\lambda t}$ for every $t \geq 0$. If $(S(t))_{t \geq 0}$ is a uniformly bounded, analytic semigroup such that $0 \in \rho(A)$, where $\rho(A)$ is the resolvent set of $A$, then it is possible to define the fractional power $(-A)^{\alpha}$ for $0<\alpha \leq 1$, as a closed linear operator on its domain $D(-A)^{\alpha}$. Furthermore, the subspace $D(-\bar{A})^{\alpha}$ is dense in $X$, and the expression

$$
\|h\|_{\alpha}=\left\|(-A)^{\alpha} h\right\|
$$

defines a norm in $D(-A)^{\alpha}$. If $X_{\alpha}$ represents the space $D(-A)^{\alpha}$ endowed with the norm $\|.\|_{\alpha}$, then the following properties hold (see [19], p. 74).

Lemma 2.5. Suppose that $A, X_{\alpha}$, and $(-A)^{\alpha}$ are as described above.

(i) For $0<\alpha \leq 1, X_{\alpha}$ is a Banach space.

(ii) If $0<\beta \leq \alpha$, then the injection $X_{\alpha} \hookrightarrow X_{\beta}$ is continuous.

(iii) For every $0<\alpha \leq 1$, there exists $M_{\alpha}>0$ such that

$$
\left\|(-A)^{\alpha} S(t)\right\| \leq M_{\alpha} t^{-\alpha} e^{-\lambda t}, \quad t>0, \quad \lambda>0 .
$$




\section{Controllability Result}

In this section we derive controllability conditions for a class of neutral stochastic functional differential equations with infinite delays driven by a fractional Brownian motion in a real separable Hilbert space. Before starting, we introduce the concepts of a mild solution of the problem (1.1) and the meaning of controllability of neutral stochastic functional differential equation.

Definition 3.1. An $X$-valued process $\{x(t): t \in(-\infty, T]\}$ is a mild solution of (1.1) if

(1) $x(t)$ is measurable for each $t>0, x(t)=\varphi(t)$ on $(-\infty, 0],\left.\Delta x\right|_{t=t_{k}}=I_{k}\left(x\left(t_{k}^{-}\right)\right)$, $k=1,2, \ldots, m$, the restriction of $x($.$) to [0, T]-\left\{t_{1}, t_{2}, \ldots, t_{m}\right\}$ is continuous, and for each $s \in[0, t)$ the function $A S(t-s) g\left(s, x_{s}\right)$ is integrable,

(2) for arbitrary $t \in[0, T]$, we have

$$
\begin{aligned}
x(t) & =S(t)(\varphi(0)-g(0, \varphi))+g\left(t, x_{t}\right) \\
& +\int_{0}^{t} A S(t-s) g\left(s, x_{s}\right) d s+\int_{0}^{t} S(t-s) f\left(s, x_{s}\right) d s \\
& +\int_{0}^{t} S(t-s) B u(s) d s+\int_{0}^{t} S(t-s) \sigma(s) d B^{H}(s), \\
& +\sum_{0<t_{k}<t} S\left(t-t_{k}\right) I_{k}\left(x\left(t_{k}^{-}\right)\right), \quad \mathbb{P}-a . s .
\end{aligned}
$$

Definition 3.2. The impulsive neutral stochastic functional differential equation (1.1) is said to be controllable on the interval $(-\infty, T]$ if for every initial stochastic process $\varphi$ defined on $(-\infty, 0]$, there exists a stochastic control $u \in L^{2}([0, T], U)$ such that the mild solution $x(\cdot)$ of $(1.1)$ satisfies $x(T)=x_{1}$, where $x_{1}$ and $T$ are the preassigned terminal state and time, respectively.

Our main result in this paper is based on the following fixed point theorem.

Theorem 3.3. (Karasnoselskii's fixed point theorem) Let $V$ be a bounded closed and convex subset of a Banach space $X$ and let $\Pi_{1}, \Pi_{2}$ be two operators of $V$ into $X$ satisfying:

(1) $\Pi_{1}(x)+\Pi_{2}(x) \in V$ whenever $x \in V$,

(2) $\Pi_{1}$ is a contraction mapping, and

(3) $\Pi_{2}$ is completely continuous.

Then, there exists $a z \in V$ such that $z=\Pi_{1}(z)+\Pi_{2}(z)$.

In order to establish the controllability of (1.1), we impose the following conditions on the data of the problem:

$(\mathcal{H} .1) A$ is the infinitesimal generator of an analytic semigroup, $(S(t))_{t \geq 0}$, of bounded linear operators on $X$. Further, $0 \in \rho(A)$, and there exist constants $M, M_{1-\beta}$ such that

$$
\|S(t)\|^{2} \leq M \quad \text { and }\left\|(-A)^{1-\beta} S(t)\right\| \leq \frac{M_{1-\beta}}{t^{1-\beta}}, \text { for all } t \in[0, T]
$$

(see Lemma 2.5).

$(\mathcal{H} .2) f$ is $L^{2}$-Carathéodory map and there exist positive constants $M_{f}, \overline{M_{f}}$ for $t \in$ $[0, T], x_{1}, x_{2} \in \mathcal{B}_{h}$ such that

$$
\mathbb{E}\left\|f\left(t, x_{1}\right)-f\left(t, x_{2}\right)\right\|^{2} \leq M_{f}\left\|x_{1}-x_{2}\right\|_{\mathcal{B}_{h}}^{2} \text {, and } \quad \overline{M_{f}}=\sup _{t \in[0, T]}\|f(t, 0)\|^{2} \text {. }
$$


$(\mathcal{H} .3)$ There exist constants $0<\beta<1, M_{g}>0$ and $\nu>0$ such that the function $g$ is $X_{\beta}$-valued and satisfies

i) $\mathbb{E}\left\|(-A)^{\beta} g(t, x)-(-A)^{\beta} g(t, y)\right\|^{2} \leq M_{g}\|x-y\|_{\mathcal{B}_{h}}^{2}, t \in[0, T]$, $x, y \in \mathcal{B}_{h}$ with $\quad \nu=4 M_{g} l^{2}\left(\left\|(-A)^{-\beta}\right\|^{2}+\frac{\left(M_{1-\beta} T^{\beta}\right)^{2}}{2 \beta-1}\right)<1$.

ii) $c_{1}=\left\|(-A)^{-\beta}\right\|$ and $\bar{M}_{g}=\sup _{t \in[0, T]}\left\|(-A)^{-\beta} g(t, 0)\right\|^{2}$.

(H.4) $I_{k}: X \longrightarrow X k=1,2, \ldots, m$, and there exist constants $M_{k} \geq 0, \widetilde{M}_{k} \geq 0$ such that $\mathbb{E}\left\|I_{k}(x)-I_{k}(y)\right\|^{2} \leq M_{k}\|x-y\|^{2}$ and $\left\|I_{k}(x)\right\|^{2} \leq \widetilde{M}_{k}$ for any $x, y \in X$.

(H.5) The function $\sigma:[0, \infty) \rightarrow \mathcal{L}_{2}^{0}(Y, X)$ satisfies

$$
\int_{0}^{T}\|\sigma(s)\|_{\mathcal{L}_{2}^{0}}^{2} d s<\infty, \quad \forall T>0 .
$$

(H.6) The linear operator $W$ from $U$ into $X$ defined by

$$
W u=\int_{0}^{T} S(T-s) B u(s) d s
$$

has an inverse operator $W^{-1}$ that takes values in $L^{2}([0, T], U) \backslash k e r W$, where

$$
\operatorname{ker} W=\left\{x \in L^{2}([0, T], U): W x=0\right\}
$$

(see [11]), and there exists finite positive constants $M_{b}, M_{w}$ such that $\|B\|^{2} \leq M_{b}$ and $\left\|W^{-1}\right\|^{2} \leq M_{w}$.

The main result of this chapter is the following.

Theorem 3.4. Suppose that $(\mathcal{H} .1)-(\mathcal{H} .6)$ hold. Then, the system (1.1) is controllable on $(-\infty, T]$ provide that

$$
7 l^{2}\left(1+8 M M_{b} M_{w} T^{2}\right)\left\{8\left(c_{1}^{2}+\frac{\left(M_{1-\beta} T^{\beta}\right)^{2}}{2 \beta-1}\right) M_{g}+8 M T^{2} M_{f}\right\}<1 .
$$

Proof. Transform the problem(1.1) into a fixed-point problem. To do this, using the hypothesis $(\mathcal{H} .6)$ for an arbitrary function $x(\cdot)$, define the control by

$$
\begin{aligned}
u(t) & =W^{-1}\left\{x_{1}-S(T)\left(\varphi(0)-g\left(0, x_{0}\right)\right)-g\left(T, x_{T}\right)\right) \\
& -\int_{0}^{T} A S(T-s) g\left(s, x_{s}\right) d s-\int_{0}^{T} S(T-s) f\left(s, x_{s}\right) d s \\
& \left.\left.-\int_{0}^{T} S(T-s) \sigma(s) d B^{H}(s)\right\}(t)-\sum_{0<t_{k}<T} S\left(T-t_{k}\right) I_{k}\left(x\left(t_{k}^{-}\right)\right)\right\}(t) .
\end{aligned}
$$

To formulate the controllability problem in the form suitable for application of the Banach fixed point theorem, put the control $u($.$) into the stochastic control system (3.3) and$ obtain a non linear operator $\Pi$ on $\mathcal{B}_{D I}$ given by

$$
\Pi(x)(t)=\left\{\begin{array}{l}
\varphi(t), \quad \text { if } t \in(-\infty, 0], \\
S(t)(\varphi(0)-g(0, \varphi))+g\left(t, x_{t}\right) \\
+\int_{0}^{t} A S(t-s) g\left(s, x_{s}\right) d s+\int_{0}^{t} S(t-s) f\left(s, x_{s}\right) d s \\
+\int_{0}^{t} S(t-s) B u(s) d s+\int_{0}^{t} S(t-s) \sigma(s) d B^{H}(s), \\
+\sum_{0<t_{k}<t} S\left(t-t_{k}\right) I_{k}\left(x\left(t_{k}^{-}\right)\right), \quad \text { if } t \in[0, T] .
\end{array}\right.
$$


Then it is clear that to prove the existence of mild solutions to equation (1.1) is equivalent to find a fixed point for the operator $\Pi$. Clearly, $\Pi x(T)=x_{1}$, which means that the control $u$ steers the system from the initial state $\varphi$ to $x_{1}$ in time $T$, provided we can obtain a fixed point of the operator $\Pi$ which implies that the system in controllable.

Let $y:(-\infty, T] \longrightarrow X$ be the function defined by

$$
y(t)= \begin{cases}\varphi(t), & \text { if } t \in(-\infty, 0], \\ S(t) \varphi(0), & \text { if } t \in[0, T],\end{cases}
$$

then, $y_{0}=\varphi$. For each function $z \in \mathcal{B}_{D I}$, set

$$
x(t)=z(t)+y(t) .
$$

It is obvious that $x$ satisfies the stochastic control system (3.3) if and only if $z$ satisfies $z_{0}=0$ and

$$
\begin{aligned}
z(t)= & \left.g\left(t, z_{t}+y_{t}\right)-S(t) g(0, \varphi)\right)+\int_{0}^{t} A S(t-s) g\left(s, z_{s}+y_{s}\right) d s \\
& +\int_{0}^{t} S(t-s) f\left(s, z_{s}+y_{s}\right) d s+\int_{0}^{t} S(t-s) B u_{z+y}(s) d s \\
& +\int_{0}^{t} S(t-s) \sigma(s) d B^{H}(s), \\
& +\sum_{0<t_{k}<t} S\left(t-t_{k}\right) I_{k}\left(z\left(t_{k}^{-}\right)+y\left(t_{k}^{-}\right)\right) .
\end{aligned}
$$

Set

$$
\mathcal{B}_{D I}^{0}=\left\{z \in \mathcal{B}_{D I}: z_{0}=0\right\}
$$

for any $z \in B_{D I}^{0}$, we have

$$
\|z\|_{\mathcal{B}_{D I}^{0}}=\left\|z_{0}\right\|_{\mathcal{B}_{h}}+\sup _{t \in[0, T]}\left(\mathbb{E}\|z(t)\|^{2}\right)^{\frac{1}{2}}=\sup _{t \in[0, T]}\left(\mathbb{E}\|z(t)\|^{2}\right)^{\frac{1}{2}} .
$$

Then, $\left(\mathcal{B}_{D I}^{0},\|\cdot\|_{\mathcal{B}_{D I}^{0}}\right)$ is a Banach space. Define the operator $\widehat{\Pi}: \mathcal{B}_{D I}^{0} \longrightarrow \mathcal{B}_{D I}^{0}$ by

$$
(\widehat{\Pi} z)(t)=\left\{\begin{array}{l}
0 \text { if } t \in(-\infty, 0] \\
\left.g\left(t, z_{t}+y_{t}\right)-S(t) g(0, \varphi)\right)+\int_{0}^{t} A S(t-s) g\left(s, z_{s}+y_{s}\right) d s \\
+\int_{0}^{t} S(t-s) f\left(s, z_{s}+y_{s}\right) d s+\int_{0}^{t} S(t-s) B u_{z+y}(s) d s \\
+\int_{0}^{t} S(t-s) \sigma(s) d B^{H}(s), \\
+\sum_{0<t_{k}<t} S\left(t-t_{k}\right) I_{k}\left(z\left(t_{k}^{-}\right)+y\left(t_{k}^{-}\right)\right), \quad \text { if } t \in[0, T]
\end{array}\right.
$$

where

$$
\begin{aligned}
u_{z+y}(t) & =W^{-1}\left\{x_{1}-S(T)\left(\varphi(0)-g\left(0, z_{0}+y_{0}\right)\right)-g\left(T, z_{T}+y_{T}\right)\right) \\
& -\int_{0}^{T} A S(T-s) g\left(s, z_{s}+y_{s}\right) d s-\int_{0}^{T} S(T-s) f\left(s, z_{s}+y_{s}\right) d s \\
& \left.\left.-\int_{0}^{T} S(T-s) \sigma(s) d B^{H}(s)\right\}(t)-\sum_{0<t_{k}<T} S\left(T-t_{k}\right) I_{k}\left(z\left(t_{k}^{-}\right)+y\left(t_{k}^{-}\right)\right)\right\}(t) .
\end{aligned}
$$

Set

$$
\mathcal{B}_{k}=\left\{z \in \mathcal{B}_{D I}^{0}:\|z\|_{\mathcal{B}_{D I}^{0}}^{2} \leq k\right\}, \quad \text { for some } k \geq 0
$$


then $\mathcal{B}_{k} \subseteq \mathcal{B}_{D I}^{0}$ is a bounded closed convex set, and for $z \in \mathcal{B}_{k}$, we have

$$
\begin{aligned}
\left\|z_{t}+y_{t}\right\|_{\mathcal{B}_{D I}} & \leq 2\left(\left\|z_{t}\right\|_{\mathcal{B}_{D I}}^{2}+\left\|y_{t}\right\|_{\mathcal{B}_{D I}}^{2}\right) \\
& \leq 4\left(l^{2} \sup _{0 \leq s \leq t} \mathbb{E}\|z(s)\|^{2}+\left\|z_{0}\right\|_{\mathcal{B}_{h}}^{2}\right. \\
& \left.+l^{2} \sup _{0 \leq s \leq t} \mathbb{E}\|y(s)\|^{2}+\left\|y_{0}\right\|_{\mathcal{B}_{h}}^{2}\right) \\
& \leq 4 l^{2}\left(k+M \mathbb{E}\|\varphi(0)\|^{2}\right)+4\|y\|_{\mathcal{B}_{h}} \\
& :=q^{\prime}
\end{aligned}
$$

From our assumptions, using the fact that $\left(\sum_{i=1}^{n} a_{i}\right)^{2} \leq n \sum_{i=1}^{n} a_{i}^{2}$ for any positive real numbers $a_{i}, i=1,2, \ldots, n$, we have

$$
\begin{aligned}
\mathbb{E}\left\|u_{z+y}\right\|^{2} \leq & 8 M_{w}\left\{\left\|x_{1}\right\|^{2}+M \mathbb{E}\|\varphi(0)\|^{2}+2 M c_{1}^{2} M_{g}\|y\|_{\mathcal{B}_{h}}^{2}\right. \\
& +2\left(c_{1}^{2}+\frac{\left(M_{1-\beta} T^{\beta}\right)^{2}}{2 \beta-1}\right)\left[M_{g} q^{\prime}+\bar{M}_{g}\right]+2 M T^{2}\left[M_{f} q^{\prime}+\overline{M_{f}}\right] \\
& \left.+2 M T^{2 H-1} \int_{0}^{T}\|\sigma(s)\|_{\mathcal{L}_{2}^{0}}^{2} d s+m M \sum_{k=1}^{m} \widetilde{M}_{k}\right\}:=\mathcal{G} .
\end{aligned}
$$

Noting that

$$
\begin{aligned}
\mathbb{E}\left\|u_{z+y}-u_{v+y}\right\|^{2} & \leq 4 M_{w}\left\{\left(c_{1}^{2}+\frac{\left(M_{1-\beta} T^{\beta}\right)^{2}}{2 \beta-1}\right) M_{g}+M T^{2} M_{f}\right. \\
& \left.+m M \sum_{k=1}^{m} M_{k}\right\}\left\|z_{t}-v_{t}\right\|_{\mathcal{B}_{h}}^{2} .
\end{aligned}
$$

It is clear that the operator $\Pi$ has a fixed point if and only if $\widehat{\Pi}$ has one, so it turns to prove that $\widehat{\Pi}$ has a fixed point. To this end, we decompose $\widehat{\Pi}$ as $\widehat{\Pi}=\Pi_{1}+\Pi_{2}$, where $\Pi_{1}$ and $\Pi_{2}$ are defined on $\mathcal{B}_{D I}^{0}$, respectively by

$$
\left(\Pi_{1} z\right)(t)=\left\{\begin{array}{l}
0 \text { if } t \in(-\infty, 0], \\
\left.g\left(t, z_{t}+y_{t}\right)-S(t) g(0, \varphi)\right)+\int_{0}^{t} A S(t-s) g\left(s, z_{s}+y_{s}\right) d s \\
\quad+\int_{0}^{t} S(t-s) \sigma(s) d B^{H}(s), \quad \text { if } t \in[0, T],
\end{array}\right.
$$

and

$$
\left(\Pi_{2} z\right)(t)=\left\{\begin{array}{l}
0 \text { if } t \in(-\infty, 0], \\
\quad \int_{0}^{t} S(t-s) f\left(s, z_{s}+y_{s}\right) d s+\int_{0}^{t} S(t-s) B u_{z+y}(s) d s \\
\quad+\sum_{0<t_{k}<t} S\left(t-t_{k}\right) I_{k}\left(z\left(t_{k}^{-}\right)+y\left(t_{k}^{-}\right)\right), \quad \text { if } t \in[0, T] .
\end{array}\right.
$$

In order to apply the Karasnoselskii fixed point theorem for the operator $\widehat{\Pi}$, we prove the following assertions:

(1) $\Pi_{1}(x)+\Pi_{2}(x) \in \mathcal{B}_{k}$ whenever $x \in \mathcal{B}_{k}$,

(2) $\Pi_{1}$ is a contraction;

(3) $\Pi_{2}$ is continuous and compact map. 
For the sake of convenience, the proof will be given in several steps.

Step 1: We claim that there exists a positive number $k$, such that $\Pi_{1}(x)+\Pi_{2}(x) \in \mathcal{B}_{k}$ whenever $x \in \mathcal{B}_{k}$. If it is not true, then for each positive number $k$, there is a function $z^{k}(.) \in \mathcal{B}_{k}$, but $\Pi_{1}\left(z^{k}\right)+\Pi_{2}\left(z^{k}\right) \notin \mathcal{B}_{k}$, that is $\mathbb{E}\left\|\Pi_{1}\left(z^{k}\right)(t)+\Pi_{2}\left(z^{k}\right)(t)\right\|^{2}>k$ for some $t \in[0, T]$. However, on the other hand, we have

$$
\begin{aligned}
k & <\mathbb{E}\left\|\Pi_{1}\left(z^{k}\right) \Pi_{2}\left(z^{k}\right)(t)\right\|^{2} \\
& \leq 7\left\{2 M c_{1}^{2}\left(M_{g}\|y\|_{\mathcal{B}_{h}}^{2}+\bar{M}_{g}\right)+2\left(c_{1}^{2} q^{\prime}+\bar{M}_{g}\right)+2 \frac{\left(M_{1-\beta} T^{\beta}\right)^{2}}{2 \beta-1}\left[M_{g} q^{\prime}+\bar{M}_{g}\right]\right. \\
& \left.+M M_{b} T^{2} \mathcal{G}+2 M T^{2}\left(M_{f} q^{\prime}+\overline{M_{f}}\right)+2 M T^{2 H-1} \int_{0}^{T}\|\sigma(s)\|_{\mathcal{L}_{2}^{0}}^{2} d s+M \sum_{k=1}^{m} \widetilde{M}_{k}\right\} \\
& \leq 7\left(1+8 M M_{b} M_{w} T^{2}\right)\left\{2 M c_{1}^{2}\left(M_{g}\|y\|_{\mathcal{B}_{h}}^{2}+\bar{M}_{g}\right)+2\left(c_{1}^{2}+\frac{\left(M_{1-\beta} T^{\beta}\right)^{2}}{2 \beta-1}\right)\left[M_{g} q^{\prime}+\bar{M}_{g}\right]\right) \\
& \left.+2 M T^{2}\left[M_{f} q^{\prime}+\overline{M_{f}}\right]+2 M T^{2 H-1} \int_{0}^{T}\|\sigma(s)\|_{\mathcal{L}_{2}^{0}}^{2} d s+m M \sum_{k=1}^{m} \widetilde{M}_{k}\right\} \\
& +8 M M_{b} M_{w} T^{2}\left(\left\|x_{1}\right\|^{2}+M \mathbb{E}\|\varphi(0)\|^{2}\right) \\
& \left.\leq \bar{K}+7\left(1+8 M M_{b} M_{w} T^{2}\right)\left\{c_{1}^{2}+2 \frac{\left(M_{1-\beta} T^{\beta}\right)^{2}}{2 \beta-1}\right) M_{g} q^{\prime}+2 M T^{2} M_{f} q^{\prime}\right\},
\end{aligned}
$$

where $\bar{K}$ is independent of $k$. Dividing both sides by $k$ and taking the limit as $k \longrightarrow \infty$, we get

$$
7 l^{2}\left(1+8 M M_{b} M_{w} T^{2}\right)\left\{8\left(c_{1}^{2}+\frac{\left(M_{1-\beta} T^{\beta}\right)^{2}}{2 \beta-1}\right) M_{g}+8 M T^{2} M_{f}\right\} \geq 1 .
$$

This contradicts (3.2). Hence for some positive $k$,

$$
\left(\Pi_{1}+\Pi_{2}\right)\left(\mathcal{B}_{k}\right) \subseteq \mathcal{B}_{k} .
$$

Step 2: $\Pi_{1}$ is a contraction. Let $t \in[0, T]$ and $z^{1}, z^{2} \in \mathcal{B}_{D I}^{0}$

$$
\begin{aligned}
\mathbb{E}\left\|\left(\Pi_{1} z^{1}\right)(t)-\left(\Pi_{1} z^{2}\right)(t)\right\|^{2} & \leq 2 \mathbb{E}\left\|g\left(t, z_{t}^{1}+y_{t}\right)-g\left(t, z_{t}^{2}+y_{t}\right)\right\|^{2} \\
& +2 \mathbb{E}\left\|\int_{0}^{t} A S(t-s)\left(g\left(s, z_{s}^{1}+y_{s}\right)-g\left(s, z_{s}^{2}+y_{s}\right)\right)\right\|^{2} \\
& \leq 2 M_{g}\left\|(-A)^{-\beta}\right\|^{2}\left\|z_{s}^{1}-z_{s}^{2}\right\|_{\mathcal{B}}^{2} \\
& +2 T \int_{0}^{t} \frac{M_{1-\beta}^{2}}{(t-s)^{2(1-\beta)}} M_{g}\left\|z_{s}^{1}-z_{s}^{2}\right\|_{\mathcal{B}}^{2} \\
& \leq 2 M_{g}\left\{\left\|(-A)^{-\beta}\right\|^{2}+\frac{\left(M_{1-\beta} T^{\beta}\right)^{2}}{(2 \beta-1)}\right\}\left(2 l^{2} \sup _{0 \leq s \leq T}\right. \\
& \mathbb{E}\left\|z^{1}(s)-z^{2}(s)\right\|^{2}+2\left(\left\|z_{0}^{1}\right\|_{\mathcal{B}}^{2}+\left\|z_{0}^{2}\right\|_{\mathcal{B}}^{2}\right) \\
& \left.\leq \nu \sup _{0 \leq s \leq T} \mathbb{E}\left\|z^{1}(s)-z^{2}(s)\right\|^{2}\right) \quad\left(\text { since } z_{0}^{1}=z_{0}^{2}=0\right)
\end{aligned}
$$

Taking supremum over $t$,

$$
\left\|\left(\Pi_{1} z^{1}\right)(t)-\left(\Pi_{1} z^{2}\right)(t)\right\|_{\mathcal{B}_{D I}^{0}} \leq \nu\left\|z^{1}-z^{2}\right\|_{\mathcal{B}_{D I}^{0}},
$$


where

$$
\nu=4 M_{g} l^{2}\left(\left\|(-A)^{-\beta}\right\|^{2}+\frac{\left(M_{1-\beta} T^{\beta}\right)^{2}}{2 \beta-1}\right)<1 .
$$

Thus $\Pi_{1}$ is a contraction on $\mathcal{B}_{D I}^{0}$.

Step 3: $\Pi_{2}$ is completely continuous $\mathcal{B}_{D I}^{0}$.

(1) $\Pi_{2}$ is continuous on $\mathcal{B}_{D I}^{0}$.

Let $z^{n}$ be a sequence such that $z^{n} \longrightarrow z$ in $\mathcal{B}_{D I}^{0}$. Then, there exists a number $k>0$ such that $\left\|z^{n}(t)\right\| \leq k$, for all $n$ and a.e. $t \in[0, T]$, so $z^{n} \in \mathcal{B}_{k}$ and $z \in \mathcal{B}_{k}$. In view of 3.6 , we have

$$
\begin{aligned}
\mathbb{E}\left\|u_{z+y}-u_{v+y}\right\|^{2} & \leq 8 k M_{w}\left\{\left(c_{1}^{2}+\frac{\left(M_{1-\beta} T^{\beta}\right)^{2}}{2 \beta-1}\right) M_{g}+M T^{2} M_{f}\right. \\
& \left.+m M \sum_{k=1}^{m} M_{k}\right\} .
\end{aligned}
$$

By hypothesis $H 1-H 4$, we have

(i) $I_{k}, k=1,2, \ldots, m$ is continuous.

(ii) $f\left(t, z_{t}^{n}+y_{t}\right) \longrightarrow f\left(t, z_{t}+y_{t}\right)$ for each $t \in[0, T]$.

(iii) $g\left(t, z_{t}^{n}+y_{t}\right) \longrightarrow g\left(t, z_{t}+y_{t}\right)$ for each $t \in[0, T]$.

(iv) $A S(T-s) g\left(s, z_{s}^{n}+y_{s}\right) \longrightarrow A S(T-s) g\left(s, z_{s}+y_{s}\right)$ for each $s \in[0, T]$.

We have by the dominated convergence theorem,

$$
\begin{aligned}
\mathbb{E}\left\|\left(\Pi_{2} z\right)^{n}(t)-\left(\Pi_{2} z\right)(t)\right\|^{2} & \leq 3\left\{\mathbb{E}\left\|\int_{0}^{t} S(t-s) B\left[z^{n}(s)-z(s)\right] d s\right\|^{2}\right. \\
& +\mathbb{E}\left\|\int_{0}^{t} S(t-s)\left[f\left(s, z_{s}^{n}+y_{s}\right)-f\left(s, z_{s}+y_{s}\right)\right] d s\right\|^{2} \\
& \left.+\mathbb{E}\left\|\sum_{0 \leq t_{k} \leq t} S\left(t-t_{k}\right)\left[I_{k}\left(z^{n}\left(t_{k}^{-}\right)+y\left(t_{k}^{-}\right)\right)-I_{k}\left(z\left(t_{k}^{-}\right)+y\left(t_{k}^{-}\right)\right)\right]\right\|^{2}\right\} \\
& \leq 3 M M_{b} T \int_{0}^{t} \mathbb{E}\left\|z^{n}(s)-z(s)\right\|^{2} d s \\
& +3 M T \int_{0}^{t} \mathbb{E}\left\|f\left(s, z_{s}^{n}+y_{s}\right)-f\left(s, z_{s}+y_{s}\right)\right\|^{2} d s \\
& +3 m M \sum_{k=1}^{m} \mathbb{E}\left\|I_{k}\left(z^{n}\left(t_{k}^{-}\right)+y\left(t_{k}^{-}\right)\right)-I_{k}\left(z\left(t_{k}^{-}\right)+y\left(t_{k}^{-}\right)\right)\right\|^{2} \\
& \longrightarrow 0 \text { as } n \longrightarrow \infty .
\end{aligned}
$$

Thus, $\Pi_{2}$ is continuous. 
(2) $\Pi_{2}$ maps $\mathcal{B}_{k}$ into equicontinuous family.

Let $z \in \mathcal{B}_{k}$ and $\tau_{1}, \tau_{2} \in[0, T], \tau_{1}, \tau_{2} \neq t_{k}, \mathrm{k}=1, \ldots, \mathrm{m}$, we have

$$
\begin{aligned}
\mathbb{E}\left\|\left(\Pi_{2} z\right)\left(\tau_{2}\right)-\left(\Pi_{2} z\right)\left(\tau_{1}\right)\right\|^{2} \leq \quad & 6 \mathbb{E}\left\|\int_{0}^{\tau_{1}}\left(S\left(\tau_{2}-s\right)-S\left(\tau_{1}-s\right)\right) f\left(s, z_{s}+y_{s}\right) d s\right\|^{2} \\
& +6 \mathbb{E}\left\|\int_{0}^{\tau_{1}}\left(S\left(\tau_{2}-s\right)-S\left(\tau_{1}-s\right)\right) B u(s) d s\right\|^{2} \\
& +6 \mathbb{E}\left\|\sum_{0<t_{k}<\tau_{1}}\left(S\left(\tau_{2}-t_{k}\right)-S\left(\tau_{1}-t_{k}\right)\right) I_{k}\left(z\left(t_{k}^{-}\right)+y\left(t_{k}^{-}\right)\right)\right\|^{2} \\
& +6 \mathbb{E}\left\|\int_{\tau_{1}}^{\tau_{2}} S\left(\tau_{2}-s\right) f\left(s, z_{s}+y_{s}\right) d s\right\|^{2} \\
& \left.+6 \mathbb{E} \| \int_{\tau_{1}}^{\tau_{2}} S\left(\tau_{2}-s\right)\right) B u(s) d s \|^{2} \\
& +6 \mathbb{E}\left\|\sum_{\tau_{1}<t_{k}<\tau_{2}} S\left(\tau_{2}-t_{k}\right) I_{k}\left(z\left(t_{k}^{-}\right)+y\left(t_{k}^{-}\right)\right)\right\|^{2} .
\end{aligned}
$$

By the inequality 3.5 and Hölder inequality, we get

$$
\begin{aligned}
\mathbb{E}\left\|\left(\Pi_{2} z\right)\left(\tau_{2}\right)-\left(\Pi_{2} z\right)\left(\tau_{1}\right)\right\|^{2} \leq \quad & 6 T \mathbb{E}\left\|\int_{0}^{\tau_{1}}\right\| S\left(\tau_{2}-s\right)-S\left(\tau_{1}-s\right) \|^{2} h_{q^{\prime}}(s) d s \\
& +6 T M_{b} \mathcal{G} \int_{0}^{\tau_{1}}\left\|S\left(\tau_{2}-s\right)-S\left(\tau_{1}-s\right)\right\|^{2} d s \\
& +6 m \sum_{0<t_{k}<\tau_{1}}\left\|S\left(\tau_{2}-t_{k}\right)-S\left(\tau_{1}-t_{k}\right)\right\|^{2} \widetilde{M}_{k} \\
& +6 T \int_{\tau_{1}}^{\tau_{2}}\left\|S\left(\tau_{2}-s\right)\right\|^{2} h_{q^{\prime}}(s) d s+6 T M_{b} \mathcal{G} \int_{\tau_{1}}^{\tau_{2}}\left\|S\left(\tau_{2}-s\right)\right\|^{2} d s \\
& +6 M m \sum_{\tau_{1}<t_{k}<\tau_{2}} \widetilde{M}_{k} .
\end{aligned}
$$

The right-hand side is independent of $z \in \mathcal{B}_{k}$ and tends to zero as $\tau_{2}-\tau_{1} \longrightarrow 0$, since the compactness of $S(t)$ for $t>0$ implies the continuity in the uniform operator topology. Thus, $\Pi_{2}$ maps $\mathcal{B}_{k}$ into an equicontinuous family of functions. The equicontinuities for the cases $\tau_{1}<\tau_{2} \leq 0$ and $\tau_{1}<0<\tau_{2}$ are obvious.

(3) $\left(\Pi \mathcal{B}_{q}\right)(t)$ is precompact set in $X$.

Let $0<t \leq T$ be fixed, $0<\epsilon<t$, for $z \in \mathcal{B}_{k}$, we define

$$
\begin{aligned}
\left(\Pi_{2, \epsilon} z\right)(t)= & S(\epsilon) \int_{0}^{t-\epsilon} S(t-s-\epsilon) f\left(s, z_{s}+y_{s}\right) d s+S(\epsilon) \int_{0}^{t-\epsilon} S(t-s-\epsilon) B u(s) d s \\
& +S(\epsilon) \sum_{0<t_{k}<t-\epsilon} S\left(t-t_{k}-\epsilon\right) I_{k}\left(z\left(t_{k}^{-}\right)+y\left(t_{k}^{-}\right)\right) .
\end{aligned}
$$

Using the estimation (3.5) as above and by the compactness of $S(t)(t>0)$, we obtain $V_{\epsilon}(t)=\left\{\left(\Pi_{2, \epsilon} z\right)(t): z \in \mathcal{B}_{k}\right\}$ is relative compact in $X$ for every $\epsilon$, 
$0<\epsilon<t$. Moreover, for every $z \in \mathcal{B}_{k}$, we have

$$
\begin{aligned}
\left.\left.\mathbb{E} \| \Pi_{2} z\right)(t)-\Pi_{2, \epsilon} z\right)(t) \|^{2} \leq & 3 T \int_{t-\epsilon}^{t}\|S(t-s)\|^{2} \mathbb{E}\left\|f\left(s, z_{s}+y_{s}\right)\right\|^{2} d s \\
& +3 T M_{b} \mathcal{G} \int_{t-\epsilon}^{t}\|S(t-s)\|^{2} d s \\
& +3 m \sum_{t-\epsilon<t_{k}<t}\left\|S\left(t-t_{k}\right)\right\|^{2} \mathbb{E}\left\|I_{k}\left(z\left(t_{k}^{-}\right)+y\left(t_{k}^{-}\right)\right)\right\|^{2} \\
\leq & 3 T M \int_{t-\epsilon}^{t} h_{q^{\prime}}(s) d s+3 T M_{b} \mathcal{G} M \epsilon \\
& +3 m M \sum_{t-\epsilon<t_{k}<t} \widetilde{M}_{k} .
\end{aligned}
$$

Therefore,

$$
\left.\left.\mathbb{E} \| \Pi_{2} z\right)(t)-\Pi_{2, \epsilon} z\right)(t) \|^{2} \longrightarrow 0, \quad \text { as } \epsilon \longrightarrow 0^{+},
$$

and there are precompact sets arbitrarily close to the set $V(t)=\left\{\left(\Pi_{2} z\right)(t): z \in\right.$ $\left.B_{k}\right\}$, hence the set $V(t)$ is also precompact in $X$.

Thus, by Arzela-Ascoli theorem $\Pi_{2}$ is a compact operator. These arguments enable us to conclude that $\Pi_{2}$ is completely continuous, and by the fixed point theorem of Karasnoselskii there exists a fixed point $z\left(\right.$.) for $\widehat{\Pi}$ on $\mathcal{B}_{k}$. If we define $x(t)=z(t)+y(t),-\infty<t \leq T$, it is easy to see that $x($.$) is a mild solution of$ (1.1) satisfying $x_{0}=\varphi, x(T)=x_{1}$. Then the proof is complete.

\section{EXAMPLE}

To illustrate the previous result, we consider the following impulsive neutral stochastic partial differential equation with infinite delays, driven by a fractional Brownian motion of the form

$$
\left\{\begin{array}{l}
\frac{\partial}{\partial t}\left[v(t, \xi)-\int_{-\infty}^{0} T(v(\theta, \xi)) G(t, \xi, \theta-t) d \theta\right]=\left[\frac{\partial^{2}}{\partial^{2} \xi} v(t, \xi)+c(\xi) u(t)\right. \\
\left.+\int_{-\infty}^{0} Q(v(\theta, \xi)) F(t, \xi, \theta-t) d \theta\right]+\sigma(t) \frac{d B^{H}(t)}{d t}, \quad 0 \leq t \leq T, t \neq t_{k}, 0 \leq \xi \leq \pi \\
\Delta v\left(t_{k}, \xi\right)=\int_{-\infty}^{t_{k}} \alpha_{k}\left(t_{k}^{-}-s\right) r(v(s, \xi)) d s, \quad k=1,2, \ldots, m ; \\
v(t, 0)=v(t, \pi)=0, \quad 0 \leq t \leq T, \\
v(s, \xi)=\varphi(s, \xi), ;-\infty<s \leq 0 \quad 0 \leq \xi \leq \pi,
\end{array}\right.
$$

where $B^{H}(t)$ is cylindrical fractional Brownian motion, $\varphi:[-\tau, 0] \times[0, \pi] \longrightarrow \mathbb{R}$ is a given continuous function such that $\varphi(s,.) \in L^{2}([0, \pi])$ is measurable and satisfies $\mathbb{E}\|\varphi\|^{2}<\infty$.

We rewrite 4.1) into abstract form of (1.1). We take $X=Y=U=L^{2}([0, \pi])$. Define the operator $A: D(A) \subset X \longrightarrow X$ given by $A=\frac{\partial^{2}}{\partial^{2} \xi}$ with

$$
D(A)=\left\{y \in X: y^{\prime} \text { is absolutely continuous, } y^{\prime \prime} \in X, \quad y(0)=y(\pi)=0\right\},
$$

then we get

$$
A x=\sum_{n=1}^{\infty} n^{2}<x, e_{n}>_{X} e_{n}, \quad x \in D(A),
$$

where $e_{n}:=\sqrt{\frac{2}{\pi}} \sin n x, n=1,2, \ldots$ is an orthogonal set of eigenvector of $-A$. 
The bounded linear operator $(-A)^{\frac{3}{4}}$ is given by

$$
(-A)^{\frac{3}{4}} x=\sum_{n=1}^{\infty} n^{\frac{3}{2}}<x, e_{n}>_{X} e_{n}
$$

with domain

$$
D\left((-A)^{\frac{3}{4}}\right)=X_{\frac{3}{4}}=\left\{x \in X, \sum_{n=1}^{\infty} n^{\frac{3}{2}}<x, e_{n}>_{X} e_{n} \in X\right\}, \text { and }\left\|(-A)^{\frac{3}{4}}\right\|=1 .
$$

It is well known that $A$ is the infinitesimal generator of an analytic semigroup $\{S(t)\}_{t \geq 0}$ in $X$, and is given by (see [19])

$$
S(t) x=\sum_{n=1}^{\infty} e^{-n^{2} t}<x, e_{n}>e_{n}
$$

for $x \in X$ and $t \geq 0$. Since the semigroup $\{S(t)\}_{t \geq 0}$ is analytic, there exists a constant $M>0$ such that $\|S(t)\|^{2} \leq M$ for every $t \geq 0$. In other words, the condition ( $\left.\mathcal{H} .1\right)$ holds.

Further for the operator $B: U \longrightarrow X$ is a bounded linear operator defined by $B u(t)(\xi)=$ $c(\xi) u(t), \quad 0 \leq \xi \leq \pi, u \in L^{2}([0, T], U)$, and the operator $W: L^{2}([0, T], U) \longrightarrow X$ given by

$$
W u(\xi)=\int_{0}^{T} S(T-s) c(\xi) u(t) d s, \quad 0 \leq \xi \leq \pi,
$$

$W$ is a bounded linear operator but not necessarily one-to-one. Let

$$
\operatorname{Ker} W=\left\{x \in L^{2}([0, T], U), W x=0\right\}
$$

be the null space of $W$ and $[K e r W]^{\perp}$ be its orthogonal complement in $L^{2}([0, T], U)$. Let $\widetilde{W}$ : $[\operatorname{Ker} W]^{\perp} \longrightarrow \operatorname{Range}(W)$ be the restriction of $W$ to $[\operatorname{Ker} W]^{\perp}, \widetilde{W}$ is necessarily one-to-one operator. The inverse mapping theorem says that $\widetilde{W}^{-1}$ is bounded since $[\operatorname{Ker} W]^{\perp}$ and Range $(W)$ are Banach spaces. So that $W^{-1}$ is bounded and takes values in $L^{2}([0, T], U) \backslash \operatorname{Ker} W$, hypothesis $(\mathcal{H} .6)$ is satisfied.

We choose the phase function $h(s)=e^{4 s}, s<0$, then $l=\int_{-\infty}^{0} h(s) d s=\frac{1}{4}<\infty$, and the abstract phase space $\mathcal{B}_{h}$ is Banach space with the norm

$$
\|\varphi\|_{\mathcal{B}_{h}}=\int_{-\infty}^{0} h(s) \sup _{\theta \in[s, 0]}\left(\mathbb{E}\|\varphi(\theta)\|^{2}\right)^{\frac{1}{2}} d s .
$$

To rewrite the initial-boundary value problem 4.1 in the abstract form we assume the following: For $(t, \varphi) \in[0, T] \times \mathcal{B}_{h}$, where $\varphi(\theta)(\xi)=\varphi(\theta, \xi),(\theta, \xi) \in(-\infty, 0] \times[0, \pi]$, we put $v(t)(\xi)=$ $v(t, \xi)$

$$
\begin{aligned}
& g(t, \varphi)(\xi)=\int_{-\infty}^{0} T(\varphi(\theta)(\xi)) G(t, \xi, \theta-t) d \theta, \\
& f(t, \varphi)(\xi)=\int_{-\infty}^{0} Q(\varphi(\theta)(\xi)) F(t, \xi, \theta-t) d \theta .
\end{aligned}
$$

The above equation can be written in the abstract form 1.1.

We now assume that the functions $\alpha_{k}: \mathbb{R} \longrightarrow \mathbb{R}, k=1,2, \ldots, m$ are continuous and $\widetilde{M}_{k}=$ $\int_{-\infty}^{t_{k}} h(s) \alpha_{k}^{2}(s) d s<\infty$. Then the condition (H.4) is satisfied if $r($.$) is Lipschitz continuous. To$ verify the conditions $(\mathcal{H} .2)$ and $(\mathcal{H} .3)$, we suppose further that

(i) the function $F(t, \xi, \theta)$ is continuous in $[0, T] \times[0, \pi] \times(-\infty, 0]$ and satisfies

$$
\int_{0}^{\pi}\left(\int_{-\infty}^{0}|F(t, \xi, \theta)| d \theta\right)^{2}<\infty
$$

(ii) the function $Q($.$) is continuous and \mathbb{E} Q^{2}(\varphi(\theta)(\xi)) \leq\|\varphi\|_{\mathcal{B}_{h}}^{2}$, for all $(\theta, \xi) \in(-\infty, 0] \times$ $[0, \pi]$. 
(iii) the function $Q($.$) is continuous and \mathbb{E}\left\|Q\left(\varphi_{1}(\theta)(\xi)\right)-Q\left(\varphi_{2}(\theta)(\xi)\right)\right\|^{2} \leq\left\|\varphi_{1}-\varphi_{2}\right\|_{\mathcal{B}_{h}}^{2}$.

We can see from $(i)$ and $(i i)$ that

$$
\mathbb{E}\|F(t, \varphi)\|^{2} \leq \int_{0}^{\pi}\left(\int_{-\infty}^{0}|F(t, \xi, \theta)| d \theta\right)^{2} d \xi\|\varphi\|_{\mathcal{B}_{h}}^{2},
$$

which implies that $(\mathcal{H} .2)$ is satisfied. Moreover

and

$$
g\left([0, T] \times \mathcal{B}_{h}\right) \subseteq D\left((-A)^{\frac{3}{4}}\right)
$$

$$
\mathbb{E}\left\|(-A)^{\frac{3}{4}} g\left(t, \varphi_{1}\right)-(-A)^{\frac{3}{4}} g\left(t, \varphi_{2}\right)\right\|^{2} \leq M_{g}\left\|\varphi_{1}-\varphi_{2}\right\|_{\mathcal{B}_{h}}^{2},
$$

for some constant $M_{g}$ depending on $T$ and $G$. Further, other assumptions are satisfied such that

$$
\left.\frac{7}{2}\left(1+8 M M_{b} M_{w} T^{2}\right)\left\{\left(1+2 M_{\frac{1}{4}}^{2} T^{\frac{3}{2}}\right)\right) M_{g}+M T^{2} M_{f}\right\}<1
$$

In order to define the operator $Q: Y:=L^{2}([0, \pi], \mathbb{R}) \longrightarrow Y$, we choose a sequence $\left\{\lambda_{n}\right\}_{n \in \mathbb{N}} \subset$ $\mathbb{R}^{+}$, set $Q e_{n}=\lambda_{n} e_{n}$, and assume that

$$
\operatorname{tr}(Q)=\sum_{n=1}^{\infty} \sqrt{\lambda_{n}}<\infty .
$$

Define the fractional Brownian motion in $Y$ by

$$
B^{H}(t)=\sum_{n=1}^{\infty} \sqrt{\lambda_{n}} \beta^{H}(t) e_{n},
$$

where $H \in\left(\frac{1}{2}, 1\right)$ and $\left\{\beta_{n}^{H}\right\}_{n \in \mathbb{N}}$ is a sequence of one-dimensional fractional Brownian motions mutually independent. Let us assume the function $\sigma:[0,+\infty) \rightarrow \mathcal{L}_{2}^{0}\left(L^{2}([0, \pi]), L^{2}([0, \pi])\right)$ satisfies

$$
\int_{0}^{T}\|\sigma(s)\|_{\mathcal{L}_{2}^{0}}^{2} d s<\infty, \quad \forall T>0 .
$$

Then all the assumptions of Theorem 3.4 are satisfied. Therefore, we conclude that the system (4.1) is controllable on $(-\infty, T]$.

\section{REFERENCES}

[1] A. Anguraj, A. Vinodkumar. Existence, uniqueness and stability results of impulsive stochastic semilinear neutral functional differential equations with infinite delays, Electron. J. Qual. Theory Differ. Equ. Vol. 2009, No. 67 (2009), 1-13.

[2] B. Boufoussi and S. Hajji, Functional differential equations driven by a fractional Brownian motion, Computers and Mathematics with Applications, 62 (2011), 746-754.

[3] B. Boufoussi, S. Hajji, and E. Lakhel, Functional differential equations in Hilbert spaces driven by a fractional Brownian motion, Afrika Matematika, 23 (2) (2012), 173-194.

[4] B. Boufoussi and S. Hajji, Neutral stochastic functional differential equation driven by a fractional Brownian motion in a Hilbert space, Statist. Probab. Lett., 82 (2012), 1549-1558.

[5] T. A. Burton and C. A. Kirk, Fixed point theorem of Karasnoselskii-Schaefer type. Math. Nachr., 189 (1998), 23-31.

[6] T. Caraballo and M.A. Diop, Neutral stochastic delay partial functional integro-differential equations driven by a fractional Brownian motion, Frontiers of Mathematics in China, 8 (4) (2013), 745-760.

[7] T. Caraballo, M.J. Garrido-Atienza, and T. Taniguchi, The existence and exponential behavior of solutions to stochastic delay evolution equations with a fractional Brownian motion, Nonlinear Analysis, 74 (2011), 3671-3684.

[8] N. T. Dung, Stochstic Volterra integro-differential equations driven by by fractional Brownian motion in Hilbert space, Stochastics, 87 (1) (2015), 142-159.

[9] G. Goldstein and A. Jerome, Semigroups of linear operators and applications Oxford Mathematical Monographs, The Clarendon Press, Oxford University Press, New York (1985).

[10] J. K. Hale, and J. Kato, Phase space for retarded equations with infinite delay, Funkcial Ekvac., 21 (1978), $11-41$. 
[11] J. Klamka, Stochastic controllability of linear systems with delay in control, Bull. Pol. Acad. Sci. Tech. Sci., 55 (2007), 23-29.

[12] J. Klamka, Controllability of dynamical systems. A survey. Bull. Pol. Acad. Sci. Tech. Sci., 61 (2013), 221229.

[13] E. Lakhel, Controllability Of Neutral Stochastic Functional Integro-Differential Equations Driven By Fractional Brownian Motion. Stochastic Analysis and Applications (To appear).

[14] E. Lakhel and S. Hajji, Existence and Uniqueness of Mild Solutions to Neutral SFDEs driven by a Fractional Brownian Motion with Non-Lipschitz Coefficients. Journal of Numerical Mathematics and Stochastics, 7 (1) (2015), 14-29.

[15] E. Lakhel, E. and M. A. McKibben, Controllability of Impulsive Neutral Stochastic Functional IntegroDifferential Equations Driven by Fractional Brownian Motion. Chapter 8 In book : Brownian Motion: Elements, Dynamics, and Applications. Editors: M. A. McKibben \& M. Webster. Nova Science Publishers, New York, 2015, pp. 131-148.

[16] Y. Li and B. Liu Existence of solution of nonlinear neutral functional differential inclusion with infinite delay. Stoc. Anal. Appl. 25 (2007), 397-415.

[17] R. Maheswari and S. Karunanithi Asymptotic stability of stochastic impulsive neutral partial functional differential equations. International J. of comp. Appli. No 18 (2014), 23-26.

[18] D. Nualart, The Malliavin Calculus and Related Topics, second edition, Springer-Verlag, Berlin (2006).

[19] A. Pazy, Semigroups of Linear Operators and Applications to Partial Differential Equations. Applied Mathematical Sciences, vol. 44, Springer-Verlag, New York (1983).

[20] Y. Ren, X. Cheng, and R. Sakthivel, On time-dependent stochastic evolution equations driven by fractional Brownian motion in Hilbert space with finite delay, Mathematical methods in the Applied Sciences, 37 (2013), 2177-2184.

[21] Y. Ren, L. Hu, and R. Sakthivel, Controllability of impulsive neutral stochastic functional differential inclusions with infinite delay, J. Comput. Appl. Math., 235 (8) (2011), 2603-2614.

[22] R. Sakthivel, R. Ganesh, Y. Ren, and S. M. Anthoni, Approximate controllability of nonlinear fractional dynamical systems, Commun. Nonlinear Sci. Numer. Simul., 18 (2013), 3498-3508.

[23] Y. Ren, H. Dai, and R. Sakthivel. Approximate controllability of stochastic differential system driven by a Levy process, Internat. J. Control, 86 (2013), 1158-1164.

[24] R. Sakthivel, J.W. Luo. Asymptotic stability of impulsive stochastic partial differential equations, Statist. Probab. Lett., 79 (2009), 1219 - 1223.

[25] D. Xu and Z.Yang. Exponential stability of nonlinear impulsive neutral differential equations with delays, Nonlinear Anal. 67 (5) (2006), 14261439. 